\title{
A survey on hepatitis B vaccination policies in genitourinary medicine in UK and Ireland
}

\author{
A El-Dalil, K W Radcliffe, J Bailey, R A Richmond, A A H Wade
}

\begin{abstract}
Objective-To determine the policies applied in genitourinary medicine (GUM) departments in the United Kingdom (UK) and Ireland with regard to hepatitis $B$ vaccination.

Design-All genitourinary medicine consultants were sent a questionnaire requesting information concerning their selection criteria and the management of patients offered Hepatitis $B$ vaccine. If no response was obtained a second questionnaire was sent. The survey was carried out in 1993.
\end{abstract}

Setting-All genitourinary medicine departments in UK and Ireland.

Participants-234 consultants were sent the questionnaire. 153 consultants responded.

Results-Overall, there was a $65.4 \%$ response rate to the questionnaire. Almost all genitourinary physicians would offer the vaccine to male homosexuals and up to $74 \%$ offer it to all male homosexuals, intravenous drug users (IVDU) and prostitutes. Of the genitourinary physicians, $96 \%$ agreed that hepatitis $B$ virus (HBV) serological markers should be checked prior to or simultaneously with vaccination, yet there was no agreement as to which marker should be performed. Up to $79 \%$ of consultants recalled the patients to check their response to vaccination but only $61 \%$ offered long term follow up after vaccination.

Conclusion-HBV vaccine is offered to all male homosexuals, IVDU and prostitutes in GUM departments. Currently, the vaccine is not generally offered to patients who present with sexually transmitted diseases. Almost all genitourinary physicians (96\%) agree that HBV serological markers should be checked prior to or simultaneously with the start of the vaccination course and $80 \%$ would request hepatitis $B$ surface antibody levels after vaccination to identify inadequate responders and non-responders. From this survey, there appears to be a need for uniform post-vaccination $\mathrm{HBV}$ screening and timing and frequency of booster doses.

(Genitourin Med 1995;71:251-253)

Keywords: hepatitis B; vaccination; sexually transmitted disease
Introduction

Hepatitis B infection is usually a self-limited disease, yet chronic carriage of hepatitis $B$ virus (HBV) follows acute infection in a variable proportion of cases. In adults, the risk of chronicity following acute infection is less than $5 \%$ and the infection is acquired through sexual or parenteral exposure. ${ }^{1}$ Prevention and control of HBV infection is now attainable through the use of hepatitis $B$ vaccine. In the UK, this is directed towards high risk groups. This survey was designed to assess the policies amongst genitourinary physicians in UK and Ireland with regard to offering HBV vaccination in GUM departments.

\section{Methods}

A questionnaire was sent to all consultants in GUM in the UK and Ireland. If no response was obtained, a second questionnaire was sent. Information regarding the risk groups of patients who were offered the HBV vaccine, hepatitis B serological tests carried out routinely and follow-up after vaccination was gathered. Also, procedures when patients failed to complete or suboptimally responded to the vaccine were collected. Finally, whether or not GUM Departments had a written policy on $\mathrm{HBV}$ screening and vaccination.

\section{Results}

Overall, there was a $65.4 \% \quad(153 / 234)$ response rate to the questionnaire. Six questionnaires were excluded from the analysis. Three did not offer the vaccine and three were duplicate questionnaires. Of the genitourinary physicians the $54 \%(75 / 147)$ who responded had a formal policy for HBV screening and vaccination within their unit.

Of genitourinary physicians who responded $99 \cdot 3 \%(146 / 147)$ offered the vaccine to all male homosexuals, $96 \%$ (141/147) offered it to intravenous drug users (IVDU) whilst $75 \%$ $(110 / 147)$ would offer it to prostitutes. The vaccine was offered to all three risk groups by $74 \%(109 / 147)$ of the responders. Only $6 \%$ $(9 / 147)$ of genitourinary physicians offered the vaccine to heterosexual patients attending with sexually transmitted diseases (STDs). This questionnaire did not address other risk factors in heterosexual patients such as multiple sexual partners or ethnic origin.

Most genitourinary physicians (86\%) would offer the vaccine after HBV serological screening, while $10 \%$ would start the vaccination course at the same visit when HBV 
Table 1 Frequency of use of hepatitis B markers (\%)

\begin{tabular}{llr}
\hline HBS Ag & 89 & 131 \\
Anti-HBc & 52 & 77 \\
Anti-HBs & 54 & 79 \\
\hline
\end{tabular}

serology is performed. Only 6/147 (4\%) would vaccinate patients without prior $\mathrm{HBV}$ screening. Routine HBV serological markers included one or a combination of hepatitis B surface antigen (HBsAg), hepatitis B core antibody (anti-HBc) and hepatitis B surface antibody (anti-HBs). HBsAg was the commonest marker requested followed by antiHBs then anti-HBc (tables 1,2$)$.

Following vaccination, $79 \%$ of consultants recalled their patients within one to three months to check their response to vaccination. $80 \%$ of consultants considered an anti$\mathrm{HBs}$ titre of more than $100 \mathrm{miu} / \mathrm{ml}$ to be an optimal response to vaccination. Long term follow up after optimal response to vaccination was offered by $61 \%$ but only $54 \%$ specifically recommended to their patients that surface antibody titres should be repeated after three to five years. Patients with suboptimal response to vaccination were offered a single booster dose and subsequent measurement of their anti-HBs titre by $62 \%$ of consultants whilst $29 \%$ of consultants would offer up to three booster doses of the vaccine and a repeat anti-HBs when necessary. If patients failed to complete the vaccination course $80 \%$ of consultants would make efforts to contact them.

\section{Discussion}

Who should be offered the vaccine?

The Department of Health (DOH) recommended that immunisation be offered to individuals who are at increased risk of hepatitis $B$ because of their lifestyle. ${ }^{2}$ Homosexual males, IVDU and prostitutes are amongst those groups. There is also no specific recommendation to immunise patients presenting with sexually transmitted disease (STD). Seroprevalence studies in heterosexual patients attending two genitourinary clinics in the West Midlands showed that the prevalence of HBV markers is low $(1.9 \%),{ }^{3}$ thus not justifying extending the vaccination policy in those clinics to cover all heterosexual patients who frequently change partners or those who present with STD. This is in contrast with parts of Europe and the United States where such patients are offered the HBV vaccine. However, the reported prevalence of $\mathrm{HBV}$ markers in similar populations in Europe and the United States ranged from 8-30\%.4-6

According to the $\mathrm{DOH}$, prostitutes should be vaccinated. Knowing that this group of patients are reluctant to approach health care workers openly, it should be a policy to make $\mathrm{HBV}$ vaccine readily available to prostitutes in the clinics and outreach posts.

Which HBV serological marker and when?

Prevaccination hepatitis B screening should be routinely carried out for male homosexuals, IVDU and prostitutes as the prevalence of HBV infection is high in those groups. ${ }^{7} a$. Hepatitis $B$ surface antigen ( $\mathrm{HBs} A \mathrm{~g}$ ) is regarded as a primary marker of hepatitis $B$ infection. It is tested for when clinical or subclinical hepatitis B infection is suspected or when screening populations for carrier status. ${ }^{8}$ It is also worth noting that in acute delta hepatitis infection, HBsAg may become suppressed or even undetectable. ${ }^{9} b$. Hepatitis $B$ core antibody $(A n t i-H B c)$ is the best marker for epidemiological monitoring of at risk populations and of past infection, it appears in the blood shortly after HBsAg, following natural infection and remains detectable for many years and often for life.$^{8} c$. Hepatitis $B$ surface antibody (Anti-HBs) is the antibody of immunity produced in response to vaccination. However, following natural infection with hepatitis B, anti-HBs is frequently transient. It may be missed if the patient is tested too early or too late.

There is lack of agreement as to which HBV marker should be included in a prevaccination screen. This should include anti-HBc which should pick up, ideally, patients who have not been exposed to HBV and should be vaccinated. Those who are anti-HBc positive should be further tested for HBsAg to identify HBV carriers and offer them appropriate medical attention.

Postvaccination HBV screen and booster doses WHO stated $^{9}$ that quantitative evaluation of the immune response after vaccination is desirable in order to identify inadequate responders and non-responders. Anti-HBs should ideally be measured one to three months after completion of the basic course of immunisations. $80 \%$ of the genitourinary physicians followed these recommendations and considered anti-HBs titre $>100 \mathrm{miu} / \mathrm{ml}$ to be an optimal response to vaccination. An individual with a peak of anti-HBs below 10 $\mathrm{miu} / \mathrm{ml}$ after the basic course of vaccination probably lacks protection against HBV infection. ${ }^{9}$ Approximately $5 \%$ of healthy individuals under the age of 40 years and up to $50 \%$ of individuals with chronic renal failure, ${ }^{10}$ alcoholic liver disease, ${ }^{11}$ HIV positive patients ${ }^{12}$ and the elderly ${ }^{13}$ will fail to mount a sufficient anti-body response. Individuals with peak anti-HBs levels of $10-100 \mathrm{miu} / \mathrm{ml}$ (suboptimal response) generally lack detectable antiHBs within one year. Suboptimal responders (anti-HBs $10-100 \mathrm{miu} / \mathrm{ml}$ ) and non-responders (anti-HBs $<10 \mathrm{miu} / \mathrm{ml}$ ) should be considered for a booster dose or, possibly, for a repeat course of vaccine. After a good response $(>100 \mathrm{miu} / \mathrm{ml})$ to a vaccination course, the length of protection remains 
unknown. Partial immunity apparently persists also after anti-HBs has declined to undetectable levels. ${ }^{14} \mathrm{HBV}$ infections have been reported in several adults who have responded to HBV-vaccine, generally after antibody levels had become very low or undetectable, ${ }^{15}$ but seldom lead to clinical disease or a chronic carrier state. Strategies for booster doses need to be formulated. Options include: (1) no booster and reliance or immunological memory, (2) give booster doses to all vaccinated individuals at three to five year intervals or (3) test anti-HBs levels and give booster doses when antibody levels fall below 10 (IU). At present, only options (2) and (3) are justifiable and the third option may be the ideal but is also the most expensive.

\section{Conclusion}

HBV vaccine is offered to male homosexuals, IVDU and prostitutes in GUM departments. Currently, the vaccine is not generally offered to heterosexual patients who present with sexually transmitted diseases. This questionnaire did not enquire about subgroups of heterosexuals who may be at risk, for example ethnic groupings, multiple partners. Almost all genitourinary physicians (96\%) agree that HBV serological markers should be checked prior to or simultaneously with the start of the vaccination course and $80 \%$ would request anti$\mathrm{HBs}$ to identify inadequate responders and non-responders. From this survey there appears to be a need for uniform guidelines to cover areas as pre-vaccination serological testing, post vaccination HBV screening and timing and frequency of booster doses.

1 Beasley RP, Hwang L-Y, Lin CC. Incidence of hepatitis among students at the University in Taiwan. $A m$ Epidemiol 1983;117:2134-222.

2 Boag F. Hepatitis B: Heterosexual transmission and vaccination strategies. Int $\mathcal{F}$ STD AIDS 1991;2:318-24.

3 Dalil A, Wade AAH, Shahmanesh M, et al. A prospective controlled survey of Hepatitis B virus seroprevalence in patients attending two genitourinary medicine clinics in the West Midlands. Spring meeting of the MSSVD, Zurich, Switzerland 6.5.1993.

4 Alter MJ, Ahtone J, Weifuse I, et al. Hepatitis B virus transmission between heterosexuals. $¥ A M A 1988 ; 256$ : transm $130-1$.

5 Hentzer B, Skinhoj P, Hoybye G, et al. Viral hepatitis in a venereal clinic population. Scand $\mathcal{F}$ Infect Dis 1980; 12:245-9.

6 Siebke JC, Wessel N, Krandal P, et al. The prevalence of hepatitis A and B in Norwegian merchant seamenserological study. Infection 1989;17:77-80.

7 Schreeder MT, Thompson SE, Hadler SCC, et al. Hepatitis B in homosexual men: prevalence of infection and factors related to transmission. F Infect Dis 1982; 146:7-15.

8 Shattock AG. Serological perspective on hepatitis B and D. Int $\mathcal{F}$ STD AIDS 1990;1:321-7.

9 International Group Immunisation against hepatitis B. Lancet 1988;i:875-6.

10 Stevens CE, Alter HJ, Taylor PE, et al. Hepatitis B vaccine in patients receiving haemodialysis. Immunogenicity and efficacy. $N$ Engl $₹$ Med 1984;311:496-501.

11 Degos F, Duhamel G, Brechot C, et al. Hepatitis B vaccination in chronic alcoholics. $\mathcal{F}$ Hepatol 1986;2:402-9.

12 Collier AC, Core L, Murphy VL, Handsfield HH Antibody to human immunodeficiency virus (HIV) and suboptimal response to hepatitis B vaccination. $A m$ Intern Med 1988;109:102-5.

13 Denis K, Mounier M, Hessel L, et al. Hepatitis B vaccination in the elderly. $\mathcal{F}$ Infec Dis 1984;149:1019.

14 Catterall AP, Murray-Lyon IM. Strategies for hepatitis B immunisation. Gut 1992;33:576-9.

15 Hadler SC, Francis DP, Maynard JE, et al. Long term immunogenicity and efficiency of hepatitis $B$ vaccine in homosexual men. $N$ Engl f Med 1986;315:209-14. 